\title{
LINEARIZED STABILITY PRINCIPLE FOR DIFFERENTIAL EQUATIONS WITH DELAYS
}

\author{
Leonid Berezansky ${ }^{1,}{ }^{*}$, brznsky@cs.bgu.ac.il, \\ Elena Braverman ${ }^{2, ~ * *}$ \\ ${ }^{1}$ Ben-Gurion University of the Negev, Beer-Sheva, Israel, \\ ${ }^{2}$ University of Calgary, Calgary, Canada
}

\begin{abstract}
In this article a linearized global stability principle is announced for nonlinear delay differential equations which is illustrated by several models of Population Dynamics.

Is given a review of some mathematical models with possible applications of the linearized principle is presented.

Keywords: delay differential equations, linearized global stability principle.
\end{abstract}

\section{Introduction}

Many applied scientists ask the following question: why do we need to study differential equations with delays when so much is known about equations without delays and these equations are much easier? The answer is: because so many processes, both natural and man-made, in biology, medicine, chemistry, engineering, economics etc. involve time delays. Whether you like it or not, time delays occur so often in almost every situation that to ignore them means to ignore the reality.

A simple example in nature is reforestation. After cutting a forest and replanting, it will take at least 20 years before reaching any kind of maturity. Hence any mathematical model of forest harvesting should involve time delays.

Similarly any model of population dynamics without delays is an approximation at best.

Another motivation for studying DDE comes from their applications in feedback control theory. In his study of ship stabilization Minorsky (1942) pointed out very clearly the importance of incorporating delays in the feedback mechanism.

We would like to present here several applied models with time delays.

1. The model of population growth (the delay logistic equation)

$\frac{d N}{d t}=r N(t)\left[1-\frac{N(t-\tau)}{K}\right]$.

involves a constant concentrated delay. This equation was first introduced by Hutchinson in 1948.

Another equation is the logistic equation with a distributed delay

$\frac{d N}{d t}=r N(t)\left[1-\frac{N(t)}{K}-\int_{0}^{t} N(s) G(t-s) d s\right]$.

This equation was introduced in 1978 by MacDonalds.

2. Lotka-Volterra predator-prey system with distributed delays

$$
\begin{aligned}
& \dot{x}(t)=x(t)\left(a-b x(t)-\int_{-r}^{0} F_{1}(s) y(t+s) d s,\right. \\
& \dot{y}(t)=y(t)\left(-c+d y(t)-\int_{-r}^{0} F_{2}(s) x(t+s) d s,\right.
\end{aligned}
$$

was introduced in 1928.

3. Delay models in medicine allowed to describe dynamic diseases which correspond to sustainable oscillations of the following equations:

${ }^{*}$ Partially supported by the Israeli Ministry of Absorption.

${ }^{* *}$ Partially supported by the NSERC Research Grant. 
$\dot{x}(t)=a-\frac{x(t) x^{n}(t-\tau)}{b+x^{n}(t-\tau)}$,

$\dot{x}(t)=\frac{a}{b+x^{n}(t-\tau)}-c x(t)$,

$\dot{x}(t)=\frac{x(t-\tau)}{b+x^{n}(t-\tau)}-c x(t)$.

These equations were introduced by Mackey and Glass in 1979.

4. Ship stabilization model

$\ddot{x}(t)+a(t) \dot{x}(t-h)+b(t) x(t-g)=f(t)$

was introduced by Minorsky in 1942.

\section{Comparison Between Ordinary and Delay Differential Equations}

Consider a simple linear equation without delay

$\dot{x}(t)=-x(t) ; x(0)=x_{0}>0$.

This equation has exact solution $x(t)=x_{0} e^{-t}$, this solution is positive, monotone and tends to zero as $t \rightarrow \infty$.

Consider now a simple linear equation with delay

$\dot{x}(t)=-x(t-2)$.

To find a solution of this equation we need to assign an initial function instead of an initial value. Suppose that we have the following initial function

$x(t)=1 ; t \in[-2 ; 0]$.

There is no exact formula for the solution of this problem, but we can find this solution by the method of steps.

Suppose first that $t \in[0 ; 2]$, then $\dot{x}(t)=-1 ; x(0)=1$, hence $x(t)=1-t$. If we continue this process we obtain the solution which oscillates about zero and is unstable.

So the behavior of solutions for equations with delay and without delay is quite different.

Actually the behavior of solutions also depends on the value of delay. In particular, consider the equation with any constant delay

$\dot{x}(t)=-x(t-\tau)$.

If $\tau \leq \frac{1}{e}$, then the solution of this equation with the same initial function as above is positive, monotone and tends to zero. But there are also oscillating solutions of this equation.

If $\frac{\pi}{2} \geq \tau \geq \frac{1}{e}$, then all solutions for any initial function oscillate and tend to zero.

If $\tau \geq \frac{\pi}{2}$, then all solutions oscillate and the equation is unstable.

There are several important questions on the asymptotic behavior of solutions of delay differential equations. Here we consider only one property which is a global stability of the equation. What does it mean?

Consider the nondelay logistic equation

$\dot{N}(t)=r(t) N(t)\left[1-\frac{N(t)}{K}\right]$.

The carrying capacity $K$ is a positive equilibrium. It is a simple fact that for $r(t)>r_{0}>0$ this equilibrium is locally asymptotically stable. But what is more interesting, this solution attracts all positive solutions. In this case we say that $x(t)=K$ is a global attractor or that the equation is globally asymptotically stable.

\section{Nonlinear and Linear Equations}

Consider the nonlinear differential equation with several delays

$\dot{x}(t)+\sum_{k=1}^{m} f_{k}\left(t, x\left(h_{1}(t)\right), \ldots, x\left(h_{l}(t)\right)\right)=0, t \geq 0$,

where $f_{k}\left(t, u_{1}, \ldots, u_{l}\right)$ is a Carathéodory function, $f_{k}(t, 0, \ldots, 0)=0, h_{k}(t) \leq t, \lim _{t \rightarrow \infty} h_{k}(t)=\infty$. We will assume that these conditions hold for all nonlinear functions and all delays. 
Together with equation (1) we consider for each $t_{0} \geq 0$ an initial value problem

$$
\begin{aligned}
& \dot{x}(t)+\sum_{k=1}^{m} f_{k}\left(t, x\left(h_{1}(t)\right), \ldots, x\left(h_{l}(t)\right)\right)=0, t \geq t_{0}, \\
& x(t)=\varphi(t), t<t_{0}, x\left(t_{0}\right)=x_{0} .
\end{aligned}
$$

We also assume that the following hypothesis holds:

$\varphi:\left(-\infty, t_{0}\right) \rightarrow R$ is a Borel measurable bounded function.

Definition. A locally absolutely continuous function $x: R \rightarrow R$ is called a global solution of problem (2), (3), if it satisfies equation (2) for almost all $t \in\left(t_{0} ; \infty\right)$ and equalities (3) for $t \leq t_{0}$.

We assume that a global solution of (2)-(3) exists and is unique.

\subsection{Linear equations}

Consider the linear equation with several delays

$$
\dot{x}(t)+\sum_{k=1}^{m} a_{k}(t) x\left(g_{k}(t)\right)=0,
$$

which is a partial case of equation (1). We will assume that $a_{k}(t)$ and delays $g_{k}(t) \leq t$ are Lebesgue measurable essentially bounded on $[0 ; \infty)$ functions.

The following stability definition is for nonlinear equation (2); it also is applies to linear equation (4).

Definition [1]. Equation (2) is stable if for any initial point $t_{0}$ and number $\varepsilon>0$ there exists $\delta>0$ such that the inequality $\sup _{t<t_{0}}|\varphi(t)|+\left|x\left(t_{0}\right)\right|<\delta$ implies $|x(t)|<\varepsilon, t \geq t_{0}$, for the solution (2)-(3).

Equation (2) is asymptotically stable if it is stable and all solutions of (2)-(3) for any initial point $t_{0}$ tend to zero as $t \rightarrow \infty$.

The following lemmas are modifications of the results of paper [2].

Lemma 1. Suppose $\int_{0}^{\infty} \sum_{k=1}^{m}\left|a_{k}(s)\right| d s<\infty$. Then all solutions of equation (4) are bounded.

Lemma 2. Suppose $a_{k}(t) \geq 0, \int_{0}^{\infty} \sum_{k=1}^{m} a_{k}(s) d s=\infty$,

$\lim _{t \rightarrow \infty} \sup \int_{\min _{k}\left\{g_{k}(t)\right\}}^{t} \sum_{i=1}^{m} a_{i}(s) d s<1+\frac{1}{e}$.

Then equation (4) is asymptotically stable.

Consider now a linear equation in the following form

$\dot{x}(t)+\sum_{k=1}^{m} a_{k}(t) x\left(h_{k}(t)\right)+\sum_{k=1}^{m} b_{k}(t) x\left(g_{k}(t)\right)=0$.

Lemma 3. Suppose $a_{k}(t) \geq 0$,

$\int_{0}^{\infty} \sum_{k=1}^{m} a_{k}(t) d t=\infty, \lim _{t \rightarrow \infty} \sup \sum_{k=1}^{n} \int_{g_{k}(t)}^{t} \sum_{i=1}^{m} a_{i}(s) d s<\infty$,

there exists $\lambda, 0<\lambda<1$, such that

$\sum_{k=1}^{n}\left|b_{k}(t)\right|<\lambda \sum_{k=1}^{m} a_{k}(t)$. stable.

If there exists an eventually positive solution of equation (4) then equation (6) is asymptotically

Consider a partial case of equation (6) with a nondelay term

$\dot{x}(t)+a(t) x(t)+\sum_{k=1}^{n} b_{k}(t) x\left(g_{k}(t)\right)=0$.

Lemma 4. Suppose $a(t) \geq 0$,

$\int_{0}^{\infty} a(t) d t=\infty, \lim _{t \rightarrow \infty} \sup \sum_{k=1}^{n} \int_{g_{k}(t)}^{t} a(s) d s<\infty$,

there exists $\lambda, 0<\lambda<1$, such that

$\sum_{k=1}^{n}\left|b_{k}(t)\right|<\lambda a(t)$.

Then equation (9) is asymptotically stable.

\subsection{Main result}

Now we can formulate our main result. To this end consider the following condition. There exists a partition

$$
\{1,2, \ldots, m\}=I_{1} \cup I_{2} \cup I_{3},
$$

and indices $i_{k}$, such that 


$$
\begin{aligned}
& \frac{f_{k}\left(t, u_{1}, \ldots, u_{l}\right)}{u_{i_{k}}} \leq \beta_{k}(t), u_{i_{k}} \neq 0, k \in I_{1}, \\
& \alpha_{k}(t) \leq \frac{f_{k}\left(t, u_{1}, \ldots, u_{l}\right)}{u_{i_{k}}}, u_{i_{k}} \neq 0, k \in I_{2}, \\
& \alpha_{k}(t) \leq \frac{f_{k}\left(t, u_{1}, \ldots, u_{l}\right)}{u_{i_{k}}} \leq \beta_{k}(t), u_{i_{k}} \neq 0, k \in I_{3} .
\end{aligned}
$$

Theorem 1. 1. Suppose there exist essentially bounded functions $\alpha_{k}(t), \beta_{k}(t)$

such that conditions (12)-(14) hold. Suppose also that all solutions of (4) are bounded for any $a_{k}(t)$ such that

$$
a_{k}(t) \leq \beta_{k}(t), k \in I_{1} ; a_{k}(t) \geq \alpha_{k}(t), k \in I_{2} ; \alpha_{k}(t) \leq a_{k}(t) \leq \beta_{k}(t), k \in I_{3} .
$$

Then all solutions of equation (1) are bounded.

2. Suppose there exist essentially bounded functions $\alpha_{k}(t), \beta_{k}(t)$ and numbers $A, B, A<B$ such that conditions (12)-(14) hold for all $u_{k}$ satisfying the inequality $A \leq u_{k} \leq B$. Suppose also that equation (4) is asymptotically stable for any $a_{k}(t)$ for which condition (15) holds.

Then $\lim _{t \rightarrow \infty} x(t)=0$ for all solutions $x(t)$ of equation (1) such that $A \leq x(t) \leq B$.

\section{Examples}

In this section we will apply Theorem 1 to obtain global stability results for some nonlinear delay equations. It means that we will prove that all solutions or all positive solutions tend to an equilibrium as $t \rightarrow \infty$.

Such applications consist of two steps. First by Theorem 1, Part 1 we prove that all solutions of the equation are bounded. Then by the second part of Theorem 1 we prove that every bounded solution tends to zero or to another equilibrium.

Example 1. Consider the equation

$$
\dot{x}(t)=\frac{b x(g(t))}{1+x^{2}(h(t))}-a x(t),
$$

where $0 \leq|b|<a, \lim _{t \rightarrow \infty} \sup (t-g(t))<\infty$.

We have only one nonlinear term.

Denote $f\left(t, u_{1}, u_{2}\right)=-\frac{b u_{1}}{1+u_{2}^{2}}$. We have $-|b| \leq \frac{f\left(t, u_{1}, u_{2}\right)}{u_{1}} \leq|b|$.

Consider now the linear equation

$$
\dot{y}(t)=b(t) y(g(t))-a y(t),
$$

where $-|b| \leq b(t) \leq|b|<a$. There exists $\lambda, 0<\lambda<1$, such that $|b|<\lambda a$. Then $|b(t)|<\lambda a$. By Lemma 4 equation (17) is asymptotically stable. In particular all solutions of this equation are bounded. If we take $\alpha(t)=-|b|, \beta(t)=|b|$, then condition (14) is satisfied. By the first part of Theorem 1 all solution of equation (16) are bounded.

If we take again $\alpha(t)=-|b|, \beta(t)=|b|$ and two arbitrary numbers $A, B, A<B$ as a lower and an upper bounds, respectively, then condition (14) is satisfied for $A \leq u_{i} \leq B ; i=1,2$. Hence by the second part of Theorem 1 for any solution of equation (16) such that $A \leq x(t) \leq B$ we have $\lim _{t \rightarrow \infty} x(t)=0$. Since $A$ and $B$ are arbitrary numbers, then for any solution of equation (16) we have $\lim _{t \rightarrow \infty} x(t)=0$.

If equation (16) involves an arbitrary positive degree $\gamma>0$ rather than the square in the denominator

$$
\dot{x}(t)=\frac{b x(g(t))}{1+x^{\gamma}(h(t))}-a x(t),
$$

then it is a generalization of the Mackey-Glass equation [4, 5], where $g(t) \equiv h(t) \equiv t-\tau$. Since all solutions of (18) with nonnegative initial conditions and a positive initial value are positive (see, for example, Lemma 1 in [6]), then the same argument as above leads to the conclusion that if $0 \leq b<a$, then all solutions (with nonnegative initial conditions) tend to zero.

Example 2. Consider the equation

$$
\dot{x}(t)=-b\left[1+x^{2}(g(t))\right] x(t)+a x(h(t)),
$$

where $0 \leq|a|<b, \lim _{t \rightarrow \infty} \sup (t-h(t))<\infty$. Denote $f\left(t, u_{1}, u_{2}\right)=b\left(1+u_{1}^{2}\right) u_{2}$. 
We have $\frac{f\left(t, u_{1}, u_{2}\right)}{u_{2}} \geq b>0$.

Consider now the linear equation

$\dot{y}(t)=-b(t) y(t)+a y(h(t))$,

where $b(t) \geq b>0$. There exists $\lambda, 0<\lambda<1$, such that $|a|<\lambda b \leq \lambda b(t)$. By Lemma 4 equation (20) is asymptotically stable.

Hence all solutions of this equation are bounded. If we take $\alpha(t)=b$, then condition (13) is satisfied. By the first part of Theorem 1 all solutions of equation (19) are bounded.

If we take again $\alpha(t)=b$ and two arbitrary numbers $A, B, A<B$ as a lower and an upper bounds, respectively, then condition (13) is satisfied for any $A \leq u_{i} \leq B, i=1,2$. Hence by the second part of Theorem 1 for any solution of equation (19) such that $A \leq x(t) \leq B$ we have $\lim _{t \rightarrow \infty} x(t)=0$. Since $A$ and $B$ are arbitrary numbers, then for any solution of equation (19) we have $\lim _{t \rightarrow \infty} x(t)=0$.

Example 3. Consider the equation

$$
\dot{x}(t)=-a(t) \arctan (x(h(t))),
$$

where

$$
a(t) \geq 0, \int_{0}^{\infty} a(s) d s=\infty, \lim _{t \rightarrow \infty} \sup \int_{h(t)}^{t} a(s) d s<1+\frac{1}{e} .
$$

Denote $f(t, u)=a(t) \arctan u$. We have $0 \leq \frac{f(t, u)}{u} \leq a(t), u \neq 0$.

Consider now the linear equation

$$
\dot{y}(t)=-b(t) y(h(t)),
$$

where $0 \leq b(t) \leq a(t)$. By Lemmas 1 and 2 all solutions of equation (23) are bounded. If we take $\alpha(t)=0$, $\beta(t)=a(t)$, then condition (14) is satisfied. By the first part of Theorem 1 all solutions of equation (21) are bounded.

Suppose $x(t)$ is a solution of (21). Then $|x(t)| \leq H$ for some $H>0$. There exists $\alpha>0$ such that $\frac{\arctan u}{u} \geq \alpha,|u| \leq H$. Hence $0 \leq \alpha a(t) \leq \frac{f(t, u)}{u} \leq a(t),|u| \leq H$. Consider again linear equation (23), where $\alpha a(t) \leq b(t) \leq a(t)$. By Lemma 2 equation (23) is asymptotically stable.

If we take $\alpha(t)=\alpha a(t), \beta(t)=a(t)$, and as lower and an upper bounds two numbers $A=-H ; B=H$, then condition (14) is satisfied for any $-H \leq u_{i} \leq H, i=1,2$. Hence by the second part of Theorem 1 for any solution of equation (21) such that $-H \leq x(t) \leq H$ we have $\lim _{t \rightarrow \infty} x(t)=0$. Since $x(t)$ is an arbitrary solution, then for any solution of equation (21) we have $\lim _{t \rightarrow \infty} x(t)=0$.

\section{References}

1. Hale J.K., Verduyn Lunel S.M. Introduction to Functional Differential equations. Applied Mathematical Sciences, vol. 99. Springer-Verlag, New York, 1993, pp. 67-99. DOI: 10.1007/978-1-4612-4342-7

2. Berezansky L., Braverman E. New Stability Conditions for Linear Differential Equations with Several Delays, arXiv:0806.3234v1 [math.DS], June 20, 2008. 19 p. DOI:10.1155/2011/178568

3. So J.W.H., Yu J.S., Chen M.P. Asymptotic Stability for Scalar Delay Differential Equations, Funkcial. Ekvac, 1996, vol. 39, pp. 1-17.

4. Mackey M.C., Glass L. Oscillation and Chaos in Physiological Control Systems, Science, 1977, vol. 197, pp. 287-289. DOI: 10.1126/science.267326

5. Losson J., Mackey M.C., Longtin A. Solution Multistability in First order Nonlinear Differential Delay Equations, Chaos, 1993, vol. 3, no. 2, pp. 167-176. DOI: 10.1063/1.165982

6. Berezansky L., Braverman E. Mackey-Glass Equation with Variable Coefficients, Comput. Math. Appl., 2006, vol. 51, iss. 1, pp. 1-16.

Received 5 May 2017 


\title{
ЛИНЕАРИЗОВАННЫЙ ПРИНЦИП УСТОЙЧИВОСТИ ДЛЯ ДИФФЕРЕНЦИАЛЬНЫХ УРАВНЕНИЙ С ЗАДЕРЖКАМИ
}

\author{
Л. Березанский ${ }^{1}$, Е. Браверман ${ }^{2}$ \\ ${ }_{1}^{1}$ Университет им. Бен-Гуриона в Негеве, Беэр-Шева, Израиль, \\ ${ }^{2}$ Университет Калгари, Калгари, Канада
}

\begin{abstract}
Предложен линеаризованный принцип глобальной устойчивости для нелинейной задержки в дифференциальных уравнениях, которые иллюстрируются несколькими моделями динамики населения.

Приводится обзор некоторых математических моделей с возможным применением линеаризованного принципа.

Ключевые слова: дифференщиальные уравнения с задержками, линеаризованный принции глобальной устойчивости
\end{abstract}

Березанский Леонид, отделение математики, Университет им. Бен-Гуриона в Негеве, БеэрШева, Израиль; brznsky@cs.bgu.ac.il.

Браверман Елена, кафедра математики и статистики, Университет Калгари, Калгари, Канада.

Поступила в редакцию 5 мая 2017 2.

\section{ОБРАЗЕЦ ЦИТИРОВАНИЯ}

Berezansky, L. Linearized Stability Principle for Differential Equations with Delays / L. Berezansky, E. Braverman // Вестник ЮУрГУ. Серия «Компьютерные технологии, управление, радиоэлектроника». - 2017. T. 17, № 3. - C. 129-134. DOI: 10.14529/ctcr170314

\section{FOR CITATION}

Berezansky L., Braverman E. Linearized Stability Principle for Differential Equations with Delays. Bulletin of the South Ural State University. Ser. Computer Technologies, Automatic Control, Radio Electronics, 2017, vol. 17 , no. 3 , pp. 129-134. DOI: $10.14529 /$ ctcr170314 